\title{
Interpréter les noms déverbaux : quelle relation avec la structure argumentale du verbe de base ? le cas des noms en -OIR du français
}

\author{
Fiammetta Namer \\ U Nancy 2, et UMR 7118 ATILF, CNRS \& Nancy-Université \\ fiammetta.namer@univ-nancy2.fr \\ Florence Villoing \\ U Paris 8, et UMR 7023, CNRS \\ florence.villoing@u-paris10.fr
}

\section{Introduction}

Les déverbaux font l'objet de nombreuses études. L'une des questions particulièrement explorées concerne l'héritage des propriétés argumentales du verbe de base dans le but d'expliciter le comportement syntaxique des noms déverbaux (cf. par exemple, (Alexiadou, 2001; Alexiadou and Haegeman, 2007; Booij and Van Haften, 1988; Booij, 1992; Grimshaw, 1990; Oshita, 1995; Sadler and Spencer, 1998; Samvellian, 1995; Spencer, 1999; Williams, 1981)). Une facette, néanmoins, a été moins souvent explorée, celle qui concerne la formation des noms déverbaux désignant des entités et formés par la morphologie constructionnelle, mettant ainsi au jour les propriétés des règles qui portent sur les lexèmes en entrée et en sortie. C'est à cet aspect que nous nous intéressons ici avec l'étude des déverbaux nominaux en $-O I R^{1}$ qui présentent un double intérêt puisque d'un côté, ils montrent des propriétés communes à tous les noms déverbaux mais de l'autre, ils s'en éloignent quant à la construction de leur interprétation.

La question que nous nous posons est de savoir si l'interprétation d'un nom déverbal est une fonction prédictible de la règle de nominalisation, si elle dépend des propriétés du verbe de base ou si d'autres paramètres entrent en jeu. De récents travaux en morphologie constructionnelle portant sur les noms et adjectifs déverbaux du français tendent à accréditer l'hypothèse que l'interprétation d'un déverbal renvoie soit au procès qu'exprime le verbe de base soit à l'un ou plusieurs de ses actants, en fonction des contraintes posées par la règle (Corbin, 2004; Dal, 1999; Plénat, 2005; Roché, 2003). Par exemple, l'interprétation agent/instrument des noms déverbaux en -eur (Busa, 1997; Fradin \& Kerleroux, 2003b; Kerleroux, 2004) est conditionnée par la présence obligatoire d'un verbe de base agentif, alors que la fonction des adjectifs en -able est prototypiquement de modifier le nom qui correspond au patient du verbe de base (Fradin, 2003).

Notre étude des noms déverbaux suffixés en -OIR (désormais V-oir) du français s'appuie sur les éléments de deux nomenclatures, celle du TLF (désormais V-oir $\mathrm{TLF}_{\text {) }}$ et celle de La Toile (désormais V-oir ${ }_{\mathrm{W} 3}$ ).

Notre enquête porte sur les deux points suivants :

- l'interprétation des V-oir, ce qui nous amène à définir les contraintes exercées sur la sortie du patron constructionnel,

- les propriétés des verbes de base, ce qui nous conduit à nous interroger sur les contraintes exercées par ces patrons sur l'entrée ; l'objectif étant d'évaluer dans quelle mesure il est possible d'établir une corrélation entre les propriétés des verbes et les interprétations des noms. Nous prendrons en considération plusieurs aspects du sens du verbe : le nombre de ses participants sémantiques et leur valeur, le type sémantique et/ou aspectuel du verbe de base.

L’étude des noms déverbaux en -OIR nous amène aux résultats suivants. 
- nous montrerons qu'à l'image d'autres règles de construction de déverbaux, la règle de construction des noms en -OIR privilégie nettement une ou deux interprétations à côté d'autre(s) interprétations non-prototypiques ;

- mais à la différence des autres déverbaux pour lesquels il semble exister une relation bien identifiée entre la structure argumentale du verbe de base et l'interprétation du déverbal, l'interprétation des déverbaux en -OIR apparaît relativement indépendante des propriétés argumentales du verbe de base. En effet, aucune des interprétations majeures des V-oir ne semble pouvoir être corrélée à un argument syntaxique du verbe. Il semble plutôt que les V-oir soient construits sur un participant sémantique de l'événement décrit par le verbe qui se réaliserait, en syntaxe, sous la forme d'un ajout.

En revanche, en prenant appui sur des résultats obtenus à partir d'études effectuées sur environ 700 noms, nous montrerons qu'il existe un lien entre l'interprétation et les propriétés de transitivité du verbe de base, puisque l'on observe que les V-oir instrumentaux sont très majoritairement construits sur des verbes transitifs tandis que les V-oir de lieu le sont sur des verbes intransitifs. Nous remarquerons cependant que ce lien est loin de constituer une règle, mais tout au plus une large tendance.

Nous illustrerons notre démonstration à partir d'exemples divers quant au statut (attesté, néologique), au domaine (général, spécialisé) et au registre (standard, familier, argotique...).

\section{Données et recueil de données}

\subsection{Données}

Notre étude prend appui d'une part sur les noms déverbaux en -OIR attestés dans un dictionnaire multivolumes, le TLF, et d'autre part sur une analyse rigoureuse de néologismes récoltés sur La Toile. Ces deux sources de données constituent un ensemble original qui montre la grande productivité de la suffixation en -OIR; la collecte à partir d'Internet a permis de réunir plus de 400 noms qui constituent l'ensemble noté $\mathrm{V}-\mathrm{oir}_{\mathrm{W} 3}{ }^{2}$ et environ 300 à partir du TLF, rassemblés dans la liste appelée désormais V-oir ${ }_{\mathrm{TLF}}$. La supériorité de la représentation des ${\mathrm{V}-o i{ }_{\mathrm{W}} 3}_{3}$ sur celle des V-oir ${ }_{\mathrm{TLF}}$ montre la vitalité de la règle -OIR (tout du moins dans les documents issus de La Toile), contrairement à ce qu'affirmait (Dubois, 1962).

Les exemples présentés dans ce qui suit seront, en fonction des besoins, empruntés à l'un ou l'autre des ensembles V-oir ${ }_{\mathrm{TLF}}$, V-oir ${ }_{\mathrm{W} 3}$.

\subsection{Recueil des données}

Le corpus $\mathrm{V}-$-oir $_{\mathrm{W} 3}$ a été obtenu par récupération automatique de candidats néologismes vérifiant une marque formelle donnée (ici la chaîne graphique $\operatorname{oir}(e)(s)$ ). La première étape a consisté à constituer une liste de « candidats noms » construits à partir de la liste de tous les verbes du TLF qui ne servent pas déjà de base aux noms en -OIR attestés. Dans un second temps, cette liste a alimenté un moteur de recherche (en l'occurrence $\mathrm{Yahoo}^{\mathrm{TM}}$ ) sous forme de requêtes formulées automatiquement. Une fois les formes et leur contexte textuel récupérés (la constitution de cet ensemble s'est achevée le 20 décembre 2006), chaque résultat a été ensuite scrupuleusement évalué de manière à rejeter les formes qui ne correspondent pas à un déverbal.

\section{Interprétation des $\mathrm{V}$-oir (contraintes sur la sortie de la règle)}

Il est communément admis que la règle de construction de lexèmes (désormais RCL) en -OIR construit des noms qui désignent l'instrument aidant à la réalisation du procès verbal (RASOIR ${ }^{3}$ ) ou le lieu dans lequel 
celui-ci se déroule (PARLOIR), à la quasi exclusion de tout autre type sémantique (cf. (Corbin, 1987 :691), qui cite l'exception TIROIR, patient du prédicat TIRER).

Même si lieu et instrument en sont les principales interprétations (en quantité comparable, et ce pour les deux corpus, ie. plus de $90 \%$ pour $\mathrm{V}$-oir ${ }_{\mathrm{W} 3}$ et pour $\mathrm{V}$-oir $\mathrm{TLF}_{\mathrm{TL}}$ ), notre recherche montre que les noms de $\mathrm{V}$-oir ${ }_{\mathrm{TLF}}$ et $\mathrm{V}$-oir ${ }_{\mathrm{W} 3}$ présentent d'autres interprétations, relativement périphériques (section 3.2).

\subsection{Interprétations privilégiées : instrument et lieu}

Les études consacrées aux noms déverbaux en -OIR ont depuis longtemps établi que les noms en -OIR s'interprètent majoritairement comme lieux ou instruments (Meyer-Lübke, 1894; Nyrop, 1899-1930; Sammet, 1968; Schmitz and Schmitz, 1995; Dubois, 1962; Corbin, 1987).Le problème se pose de la définition et de l'identification de ces deux types sémantiques. Comme nous le rappelons infra, section 3.2 , de nombreux travaux se sont consacrés à l'élaboration des critères et des tests permettant de classer les noms déverbaux en fonction de leur interprétation agentive, patientale ou événementielle, par rapport au prédicat de base. On s'attendrait a priori à ce que des travaux analogues se soient intéressés aux interprétations instrumentale et locative d'un nom déverbal, dans le but de déterminer, si, et comment, ce nom déverbal fait référence à un lieu ou un instrument qui participe à la réalisation du procès décrit par le verbe de base. Mais finalement, peu de travaux se consacrent à l'identification des interprétations locative/instrumentale d'un nom déverbal. Deux raisons essentielles nous semblent expliquer ce manque : (i) tout d'abord, lieu et instrument intéressent peu les syntacticiens qui analysent les syntagmes instrumentaux et locatifs comme des ajouts (voir section 4.2); (ii) d'autre part, les interprétations lieu et instrument des V-oir sont souvent considérées par les morphologues comme indissociables l'une de l'autre: (Corbin, 1990:47-48) reprenant (Corbin, 1987:247-248) indique, par exemple, que les noms en -OIR possèdent une "interprétation unique sous plusieurs sens apparemment différents. [...] la distinction instrumental/locatif n'est qu'apparente [...]". De son côté, (Plénat, 2005:250) s'interroge sur la pertinence qu'il y a à distinguer "les locatifs des instrumentaux : la distinction paraît peu opératoire en morphologie, où l'acception locative n'a pas de moyens d'expression distincts (le suffixe -OIR est à la fois locatif et instrumental)".

Il est donc admis que la RCL en -OIR construit des noms de lieu et/ou d'instrument, et que leur caractérisation exacte est difficile à prévoir, voir peu souhaitable. Cet état de fait ne nous satisfait nullement, notamment parce que certaines constructions morphologiques montrent que l'indistinction instrument/lieu ne tient pas face à des déverbaux tels que les noms en -eur dont la règle est susceptible de construire une interprétation instrumentale mais jamais locative. Aussi avons nous cherché à définir les contraintes liées à la RCL-OIR. en établissant un ensemble de critères qui permettent d'identifier les interprétations nominales de lieu et d'instrument. La première étape dans cette tâche est la constitution (section 3.1.1) puis l'application au corpus (section 3.1.2) de tests permettant de distinguer, parmi les V-oir, leur valeur sémantique (locative ou instrumentale) dans un syntagme régi par V. Ensuite, nous examinerons dans quelle mesure cette interprétation de lieu ou d'instrument, quand elle est clairement identifiable, correspond à celle d'un argument logique du prédicat $\mathrm{V}$ de base (section 4.2).

\subsubsection{Instrument ou lieu ? Les tests utilisés}

Identifier un nom d'instrument ou un nom de lieu est une tâche peu aisée dans l'absolu. En effet, un nom peut dénoter un lieu dans certaines situations (comme, par exemple, le nom BOCAL dans «le poisson rouge vit dans un bocal ») et un instrument dans d'autres (le même nom BOCAL désigne un instrument dans : «Jean a tué Marie à coups de bocal sur la tête »). La définition fournie par les dictionnaires constitue quant à elle le plus souvent un indice instable, et ne peut-être exploitée. C'est ainsi que le TLF définit le nom EGOUT comme un nom d'instrument : «installation ou conduit servant à l'écoulement d'un liquide » (la mention 'servant à' indiquant la finalité de l'objet et donc sa fonction instrumentale); dans la même entrée, ce nom est également décrit comme lieu «Lieu où viennent affluer les choses, les gens les plus vils». 
En première approximation, un nom d'instrument dénote un objet concret artéfactuel (à l'exception de certains noms de parties du corps, qui désignent des instruments par métonymie, eg. MACHOIRE, voir la section 3.1.2). De ce fait, l'instrument possède deux propriétés: celle de provenir d'une création humaine, et celle d'être destiné à un usage particulier (voir (Pustejovsky, 1995: ch6, 8) pour un modèle de représentation de ces deux traits). Cependant, (Flaux and Van de Velde, $2000: 50-52$ ) fait remarquer que tous les «noms d'objets fabriqués » incluent l'existence d'une finalité dans leur définition: tous les objets artéfactuels peuvent donc s'employer comme instrument, en fonction de l'événement auquel ils prennent part. Cette caractérisation semble peu discriminante, et donc inutilisable.

Au sein des travaux de sémantique lexicale, un nom de lieu fait référence à un objet (le « site ») qui doit comporter soit un volume soit une surface dans ou sur lequel se situe l'entité déplacée (la «cible»). L'identification du nom dénotant le site se fait donc relativement à celle du nom dénotant de la cible. A la suite de (Vandeloise, 1986), différents travaux ont permis d'établir les critères d'attribution des étiquettes de cible et de site pour les objets dénotés par les noms entrant dans la construction «NP1 prep NP2 » où prep est une préposition spatiale. Par exemple, quatre traits caractérisent le site et/ou la cible reliés par la préposition « sur » (de Mulder and Flaux, 2005; Dendale and de Mulder, 1998; Vandeloise, 2000) : le site dénote une surface, la cible est plus haut que le site, il y a contact entre cible et site, le site sert de support à la cible. La notion de lieu (de site) est donc forcément relative. Pas plus que la fonction d'instrument, elle ne peut être définie dans l'absolu pour caractériser l'objet auquel un nom donné fait référence. Prenons l'exemple du nom MIROIR : l'objet désigné, en tant qu'artéfact, est un instrument; mais quand le nom intervient dans une séquence comme «la mouche est sur le miroir», il vérifie tous les traits qui font d'un miroir un site, donc un lieu; enfin quand, à l'inverse, le nom MIROIR apparaît dans la phrase «le miroir est sur la table», l'objet auquel il fait référence a toutes les caractéristiques d'une cible.

On retrouve ici l'ambiguïté pointée par D. Corbin et M. Plénat et mentionnée supra; la sémantique lexicale ne permet pas en général de choisir, pour un V-oir donné, l'interprétation (lieu ou instrument) la plus appropriée. Par conséquent, dans le but d'optimiser notre analyse, nous avons eu recours à l'utilisation de tests syntaxiques qui mettent en jeu l'emploi de V-oir dans des constructions régies par le verbe $\mathrm{V}$ de base.

Les tests que nous avons utilisés pour identifier l'interprétation des V-oir exploitent les propriétés transformationnelles ou distributionnelles des énoncés dans lesquels apparaissent le couple (V, V-oir) et proviennent ou sont inspirés de (Boons et al., 1976 :ch3; Cadiot, 1990, 1991; Gross, 1975), et (Reinhart, 2002). Ces tests sont regroupés dans le tableau Tab1. L'étiquette lieu, instrument ou lieu/instrument est attribuée à V-oir en fonction du nombre de tests auxquels ce nom répond positivement pour chaque interprétation. Le symbole $\mathrm{W}$ désigne le complément éventuel de $\mathrm{V}$.

Parmi les tests d'identification du V-oir de lieu, nous distinguons ceux qui s'adressent aux V de mouvement inhérent notés $\mathrm{V}_{\text {mvmt }}$, ou $\mathrm{V}$ de mouvement causé, notés $\mathrm{V}_{\text {mvmtC }}$ (TEST 2mvmt) et ceux qui concernent les autres types de verbes (TEST 2a', 2b', 2c') (voir (Levin and Rappaport Hovav, 1995) pour une analyse des prédicats de mouvement à direction inhérente ; voir (Goldberg, 1995; Levin, 1993; Pinker, 1989) pour une discussion sur la notion de mouvement causé ; voir (Davis and Koenig, 2000) pour une modélisation de ces types lexicaux). En effet en cas de mouvement (causé) V-oir s'interprète comme un lieu s'il désigne la destination du protagoniste du mouvement. Dans les autres cas (TEST2a'c') il ne peut s'agir que du lieu scénique où se déroule l'action décrite par V. Ce cas présuppose pour le protagoniste (exprimé par NP0) d'être dans le lieu décrit par V-oir au début, à la fin ou pendant toute la durée du procès décrit par V (Boons et al., 1976 :205). Cette condition exprimée par le (TEST 2c') distingue MANGEOIRE, qui échoue au test ( $L a$ vache mange dans la mangeoire $=1=>$ la vache est dans la mangeoire; La vache mange dans la mangeoire $<=l=>$ la vache mange quand elle est dans la mangeoire) de DORTOIR, qui le passe avec succès ( $L$ 'élève dort dans le dortoir $=>$ l'élève est dans le dortoir ; L'élève dort dans le dortoir $<=>$ l'élève dort quand il est dans le dortoir).

Parmi les tests de reconnaissance des instrumentaux, la substitution dans le TEST1c, empruntée à (Cadiot, 1991) sert à identifier les noms d'instruments prototypiques, qui autorisent l'alternance « $\mathrm{V}$ avec un V-oir / V au V-oir » (je hache ma viande avec un hachoir / je hache ma viande au hachoir). Quant au 
TEST1d, il témoigne, en particulier, de la manière dont l'instrument se substitue parfois à l'agent en position sujet (cf. section 4.2.1.1) : Max casse la vitre avec un marteau / le marteau casse la vitre.

\begin{tabular}{|c|c|}
\hline TESTS 1 instrument & TESTS 2 Lieu \\
\hline $\begin{array}{l}\text { TEST 1a } \\
\text { NP0 V W au moyen de DET V-oir } \\
\text { NP0 V W avec DET V-oir } \\
\text { Avec quoi, grâce à quoi, à l'aide de quoi, } \\
\text { au moyen de quoi est-ce que NP0 V W ? } \\
\text { Avec/grâce à/à l'aide de/au moyen de } \\
\text { DET V-oir }\end{array}$ & $\begin{array}{l}\text { TEST 2mvmt : Vmvmt ou VmvmtC } \\
\text { NP0 } V_{\text {mvmt }} \text { PrepLOC DET V-oir } \rightarrow \text { NP0 se dirige vers } \\
\text { DET V-oir } \\
\text { NP0 V } V_{\text {mvtC NP1 PrepLOC DET V-oir } \rightarrow \text { NP1 se dirige }} \\
\text { vers DET V-oir }\end{array}$ \\
\hline $\begin{array}{l}\text { TEST 1b } \\
\text { NP0 V W avec DET V-oir } \leftrightarrow \text { NP0 utilise } \\
\text { DET V-oir pour Vinf W }\end{array}$ & $\begin{array}{l}\text { TEST 2a' : autre V } \\
\text { A quel endroit est-ce que NP0 V W ? Dans/sur DET } \\
\text { V-oir }\end{array}$ \\
\hline $\begin{array}{l}\text { TEST 1c } \\
\text { NP0 V W avec DET V-oir } \leftrightarrow \text { NP0 V W } \\
\text { au V-oir }\end{array}$ & $\begin{array}{l}\text { TEST } 2 b^{\prime} \text { : autre V } \\
\text { NP0 V W PrepLOC DET V-oir } \leftrightarrow \text { NP0 V W, et cela se } \\
\text { passe PrepLOC DET V-oir. }\end{array}$ \\
\hline $\begin{array}{l}\text { TEST 1d } \\
\text { NP0 V NP1 avec DET V-oir } \rightarrow \text { DET } \\
\text { V-oir V NP1 }\end{array}$ & $\begin{array}{l}\text { TEST 2c' : autre V } \\
\text { NP0 V W PrepLOC V-oir } \rightarrow \text { NP0 est PrepLOC V-oir } \\
\text { (au début et/ou à la fin du déroulement de V) } \\
\text { NP0 V W PrepLOC DET V-oir } \leftrightarrow \text { NP0 V W quand NP0 } \\
\text { est PrepLOC DET V-oir } \leftrightarrow \text { NP0 y V W }\end{array}$ \\
\hline
\end{tabular}

Tab1 : Tests d'identification Lieu/Instrument

\subsubsection{V-oir ${ }_{\mathrm{TLF}}$ et $\mathrm{V}$-oir ${ }_{\mathrm{W} 3}$ : application des tests}

Au moyen des tests ci-dessus, la majeure partie (c'est à dire 587 sur les 639, soit 91,8\%) des noms en -OIR issus de nos deux corpus (V-oir ${ }_{\mathrm{W} 3}$ et $\mathrm{V}$-oir $\mathrm{TLF}$ ) sont interprétables comme instrument ou comme lieu. $42,4 \%$ d'entre eux sont étiquetables comme des «noms de lieu »; ils sont plus nombreux, c'est à dire 167/283 dans le TLF que sur la Toile, où ils ne sont que 82 sur 304 . Les 57,6\% restants sont interprétables comme des «noms d'instruments »: les proportions relatives à chaque source de données sont inversées, puisque les noms d'instrument sont plus nombreux dans $V$-oir ${ }_{\mathrm{W} 3}$ : 201/304 que dans V-oir ${ }_{\text {TLF }}:$ 137/283 (on pourra se reporter au tableau Tab4 pour le détail de ces données chiffrées).

Comme le montre le tableau Tab2, la décision dépend du rapport TEST1/TEST2 des réponses positives (note + ) aux tests présentés dans le tableau Tab1. L'interprétation de certains noms reste indéterminée entre lieu et instrument (cf. deux dernières lignes du tableau). Il s'agit de noms qui satisfont aux tests TEST2a'-c', et qui peuvent donc s'interpréter comme des lieux «scéniques », tout en vérifiant également les tests TEST1a et TEST1b, c'est à dire renvoyant à des instruments non prototypiques.

\begin{tabular}{|l|l|l|l|l|}
\hline Source & Exemple & TESTS instrument & $\begin{array}{l}\text { TESTS lieu } \\
\text { (TEST2mvmt } \\
\text { TEST2a'+ TEST2b' }+ \\
\text { TEST2c') }\end{array}$ & $\begin{array}{l}\text { Score (Instr } \\
\text { /Lieu) }\end{array}$ \\
\hline
\end{tabular}




\begin{tabular}{|c|c|c|c|c|}
\hline TLF & GRATTOIR & $\begin{array}{l}\text { TEST1a }+ \text {; } \\
\text { TEST1b+ ;TEST1c+; } \\
\text { TEST1d }+\end{array}$ & $\begin{array}{l}\text { TEST2a'- ; } \\
\text { TEST2b'- ; TEST2c'- }\end{array}$ & 4/0 : Instr \\
\hline TLF & MACHOIRE & $\begin{array}{l}\text { TEST1a+; TEST1b+; } \\
\text { TEST1c-; TEST1d- }\end{array}$ & $\begin{array}{l}\text { TEST2a'- ; } \\
\text { TEST2b'- ; TEST2c'- }\end{array}$ & $2 / 0:$ Instr \\
\hline W3 & ENSACHOIR $^{4}$ & $\begin{array}{l}\text { TEST1a+; TEST1b+; } \\
\text { TEST1c-; TEST1d- }\end{array}$ & $\begin{array}{l}\text { TEST2a'+; } \\
\text { TEST2b'- ; TEST2c'- }\end{array}$ & $2 / 1:$ Instr \\
\hline TLF & PARLOIR & $\begin{array}{l}\text { TEST1a- ; TEST1b- } \\
\text {;TEST1c- ; TEST1d- }\end{array}$ & $\begin{array}{l}\text { TEST2a'+ ; } \\
\text { TEST2b'+ ; TEST2c'+ }\end{array}$ & 0/3 : Lieu \\
\hline W3 & GLANDOIR $^{5}$ & $\begin{array}{l}\text { TEST1a-; TEST1b+ } \\
\text { TEST1c-; TEST1d- }\end{array}$ & $\begin{array}{l}\text { TEST2a'+ ; } \\
\text { TEST2b'+ ; TEST2c'+ }\end{array}$ & 1/3: Lieu \\
\hline W3 & POSOIR $^{6}$ & $\begin{array}{l}\text { TEST1a- ;TEST1b- ; } \\
\text { TEST1c- ; TEST1d- }\end{array}$ & TEST2+; & 0/3: Lieu \\
\hline TLF & MIROIR & $\begin{array}{l}\text { TEST1a+; TEST1b+; } \\
\text { TEST1c-; TEST1d- }\end{array}$ & $\begin{array}{l}\text { TEST2a'+; } \\
\text { TEST2b'-; TEST2c'- }\end{array}$ & $2 / 1:$ Instr \\
\hline W3 & DEPLAÇOIR $^{7}$ & $\begin{array}{l}\text { TEST1a+ ; TEST1b+; } \\
\text { TEST1c-; TEST1d- }\end{array}$ & TEST2mvmt-; & $2 / 0:$ Instr \\
\hline TLF & PLONGEOIR & $\begin{array}{l}\text { TEST1a+; TEST1b+; } \\
\text { TEST1c-; TEST1d- }\end{array}$ & TEST2 mvmt - ; & $2 / 0:$ Instr \\
\hline TLF & ÉCHAPPATOIRE & $\begin{array}{l}\text { TEST1a+; TEST1b+; } \\
\text { TEST1c-; TEST1d- }\end{array}$ & TEST2 mvmt - ; & $2 / 0:$ Instr \\
\hline W3 & ÉCOULOIR $^{8}$ & $\begin{array}{l}\text { TEST1a+ ; TEST1b+ } \\
\text { TEST1c-; TEST1d- }\end{array}$ & TEST2 mvmt + ; & $\begin{array}{l}2 / 3: \text { Lieu ou } \\
\text { Instr }\end{array}$ \\
\hline TLF & BALANÇOIRE & $\begin{array}{l}\text { TEST1a+ ; TEST1b+ } \\
\text { TEST1c-; TEST1d- }\end{array}$ & $\begin{array}{l}\text { TEST2a'+ ; } \\
\text { TEST2b'+ ; TEST2c'+ }\end{array}$ & $\begin{array}{l}2 / 3: \text { Lieu ou } \\
\text { Instr }\end{array}$ \\
\hline
\end{tabular}

Tab2 : Exemple d'application des Tests

\subsection{Interprétations périphériques, interprétations impossibles}

Bien que les noms déverbaux en -OIR s'interprètent préférentiellement comme des instruments ou des lieux, l'étude approfondie du corpus montre que d'autres interprétations sont possibles quoique rares, à savoir l'interprétation patientale (section 3.2.1) et l'interprétation causative (section 3.2.3). Il semble, en revanche, que la règle en -OIR ne permette pas de construire des noms d'agents (section 3.2.2) ni des noms de procès (section 3.2.3) ; les rares $\mathrm{V}$-oir référant à des agents ou des procès proviennent soit d'une évolution historique originale soit de dérivations sémantiques mais ne relèvent pas directement du sens associé à la règle de construction.

A la différence des interprétations instrumentale et locative pour lesquelles la littérature fournit peu de critères, les interprétations patientale, agentive, causative et processuelle sont identifiées au moyen de critères ou de tests rendus explicites par de nombreux travaux. Parmi ceux-ci, nous avons retenu ceux de (Dowty, 1991) pour identifier patientitude et agentivité, ceux de (Mithun, 1991), pour l'agentivité, ceux 
de (Van Valin and LaPolla, 1997), concernant la causativité, et ceux de (Grimshaw, 1990; Milner, 1982) et (Gross and Kiefer, 1995) pour reconnaître un nom d'événement.

\subsubsection{Patient}

L'application des critères d'identification d'un Proto-patient avancés par (Dowty, 1991) permettent de reconnaître qu'un certain nombre de noms déverbaux en -OIR, en proportion très réduite, désignent le patient du procès qu'exprime le verbe de base. Les critères auxquels sont sensibles ces V-oir sont les suivants :

(i) l'entité désignée par V-oir « subit un changement d'état». On remarque que le nouvel état mentionné dans ce test peut être :

- soit une nouvelle localisation (TIROIR, nom d'objet dont le sens construit réfère à sa propriété de subir l'action du prédicat TIRER; SAUTOIR, nom d'un long collier ou chaîne qui pend sur la poitrine et dont le sens construit réfère à sa propriété de subir l'action du prédicat SAUTER)

- soit un état indéfini (ce qui résulte de l'action d'un verbe non télique) (le nom POUSSOIR, «Bouton sur lequel on appuie pour déclencher un mécanisme» (TLF.), les néologismes GRIFFOIR : "Ce griffoir en carton contient également de l'herbe à chat à faire pousser avant ... », ASTICOTOIR, TITILLOIR : « Il lui manque, son asticotoir, son titilloir, son défouloir »)

(ii) l'entité désignée par V-oir « est affectée causativement par un autre participant sémantique »; le plus souvent, celui-ci se réalise sous la forme d'un agent qui occuperait syntaxiquement la position sujet (NP0) dans une structure «NP0 V DET V-oir» (TIROIR: Jean tire le tiroir; POUSSOIR: Jean pousse le poussoir ; GRIFFOIR : Le chat griffe le griffoir ). Cependant, le prédicat peut également être non-agentif; dans ce cas le patient correspondrait, en syntaxe, au sujet: c'est l'exemple de SAUTOIR (Patient-Anim SAUTER : le sautoir saute sur sa poitrine).

\subsubsection{Agent}

L'application des critères d'identification d'une interprétation agentive (Dowty, 1991; Mithun, 1991) sur le corpus des noms déverbaux en -OIR aboutit à un résultat négatif. Il semblerait qu'aucun V-oir ne puisse désigner l'agent du procès qu'exprime le verbe de base, en particulier parce qu'aucun ne répond aux deux premiers critères d'agentivité $(a, b)$ rappelés ci-dessous :

a. Participation volitionnelle à l'événement ou à l'état

b. Eprouve ou perçoit une sensation

c. Cause un événement ou change l'état d'un autre participant

On note cependant quelques cas de noms en -OIR qui, en situation énonciative, référent à des humains ou des groupes d'humains, et vérifient les tests ci-dessus; cependant, dans aucun de ces cas, cette valeur agentive ne semble tenir à la règle de suffixation elle-même.

C'est ce que l'on observe en particulier avec le nom AUDITOIRE, que l'on peut retrouver en position de sujet agentif et qui répond positivement aux critères a. b. et c. de (Dowty, 1991) :

[1] «l'auditoire entendit...», «l'auditoire a applaudi poliment», «l'auditoire a réalisé l'invraisemblance de mes propos » (La Toile, Google 17/04/08).

Il est fort peu probable que cet emprunt au latin auditorium, qui renvoie dans cette langue aux deux valeurs « lieu » et « assemblée d'auditeurs », tienne de nos jours son interprétation agentive de la règle de suffixation en -OIR du français. Cette interprétation serait davantage à porter au crédit de l'évolution sémantique du terme, d'autant qu'il est l'unique exemple de construction héritée du latin qui présente cette interprétation. 
ASSOMMOIR et BASSINOIRE peuvent également s'interpréter comme agents, puisqu'ils présentent des emplois où ils réfèrent à des individus (ex. [2] et [3]).

[2] ASSOMMOIR : «Quel assommoir il était en cette belle soirée. Pourquoi ne se taisait-il pas ?» (La Toile, Google 05/05/08).

[3] BASSINOIRE : «Ou bien il [Hanotaux] se dépensait en courbettes devant ChallemelLacour et Targé, puis les traitait entre deux portes de 'vieilles moules' et de bassinoires, ce qui était exagéré. » L. DAUDET, L'Entre-deux-guerres, 1915, p. 78. (TLF)

Ces emplois sont construits sur une valeur sémantique dérivée du verbe qui en fait un prédicat de type psychologique exprimant une émotion. Bien qu'ils réfèrent en contexte à des individus, ces noms ne répondent positivitement qu'au critère c. (cause) alors qu'ils échouent à l'application des critères a. (volition) et b. ; leur sens construit, du reste, correspond à celui de noms d'instruments (d'ailleurs, pour chacun de ces noms, l'interprétation première relevée par le TLF est instrumentale). Il semblerait donc que la valeur agentive qu'ils sont susceptibles de prendre en contexte ne soit pas à porter au crédit de la RCL de suffixation en -OIR, mais plutôt à un glissement sémantique favorisé par la situation d'énonciation.

\subsubsection{Cause}

Nous recensons quelques rares noms déverbaux en -OIR néologiques que l'on interprète comme la cause de l'événement décrit par le verbe de base (sans qu'ils répondent aux critères d'agentivité) et qui répondent à la définition de la Cause telle que le proposent (Van Valin and LaPolla, 1997) : une force (cause) n'ayant aucun contrôle sur ce qui est causé :

[4] REPOUSSOIR : «La fortune du FN vint précisément de cette faiblesse : le parti lepéniste était pour la gauche le repoussoir idéal, fort en apparences...» (La Toile, Google 05/05/08).

[5] TREBUCHOIR : "la création d'un établissement administratif chargé de financer la réduction du temps de travail a été, dès l'origine, un grand " trébuchoir " pour le Gouvernement. » (La Toile, Yahoo 20/12/06).

[6] ATTIROIR : « nous devons nous adapter pour ne pas être un repoussoir mais un attiroir » (La Toile, Yahoo 20/12/06).

Les cas qui relèvent de cette interprétation semblent tellement rares qu'il serait présompteux, en l'état actuel de nos recherches, d'incriminer cette interprétation aux propriétés de construction de la RCL-OIR, d'autant plus que les exemples REPOUSSOIR et ATTIROIR s'interprètent également comme des noms d'instrument (valeur première de REPOUSSOIR) ; on peut remarquer par ailleurs qu'ATTIROIR a toutes les chances d'être construit par analogie sur le lexème REPOUSSOIR, comme en témoigne l'exemple [6] reprenant un extrait de La Toile.

\subsubsection{Procès}

De même qu'elle ne construit pas de noms d'agent, la suffixation déverbale en -OIR ne semble pas apte à former des noms qui désignent le procès décrit par le verbe de base. Les V-oir ne répondent pas, en effet, au faisceau de contraintes qui définissent habituellement les noms d'événement, et dont nous reprenons ici les plus significatives (Grimshaw, 1990; Gross and Kiefer, 1995; Milner, 1982) :

- la nature des déterminants : l'emploi du déterminant un est très difficile voire exclu avec une nominalisation processive ;

- pluralisation : les nominalisations processives n'apparaissent en principe pas sous forme de pluriel ; 
- l'emploi de certains modifieurs forcent l'interprétation événementielle: fréquent, dans une heure, depuis six semaines...;

- la capacité à servir de complément au verbe procéder à.

Le nom INTERROGATOIRE, cependant, répond à quelques-uns de ces critères (cf. [7]), laissant ainsi apparaître une interprétation processive en parallèle à son interprétation résultative (cf. l'emploi des modifieurs, le plus discriminant au regard de l'autre interprétation) :

[7] «l'interrogatoire fréquent des étrangers surprend la population» «l'interrogatoire de Jean a eu lieu il y a six jours » « Tout de suite après, l'interrogatoire militaire a débuté. » (La Toile, Google 05/05/08).

Pour autant, l'interprétation processive du nom INTERROGATOIRE semble résulter de conditions particulières liées à la nominalisation ancienne de l'adjectif latin interrogatorius; cette valeur pourrait donc vraisemblablement ne pas dépendre de la RCL elle-même, mais plutôt de l'évolution de l'emploi d'adjectif à nom (à partir du latin médiéval juridique interrogatorius).

\subsection{Synthèse}

Le tableau Tab3 répertorie l'ensemble des interprétations construites par la RCL-OIR des V-oir et extraits du TLF (première colonne) et de la Toile (deuxième colonne), selon les tests présentés aux sections 3.1 à 3.3. Il illustre le premier résultat auquel a abouti notre étude. Tout d'abord, ce résultat constitue en quelque sorte la confirmation empirique d'hypothèses émises depuis longtemps en morphologie: les V-oir s'interprètent principalement comme des lieux ou des instruments. Ensuite, il se fait l'écho d'autres travaux sur les nominalisations, en montrant l'existence d'interprétations périphériques : celles de patient et de cause. Enfin, il propose un faisceau de tests, (certainement perfectibles) aboutissant à une interprétation non ambiguë des valeurs de « lieu » et d' « instrument », pour la majeur partie des V-oir.

\begin{tabular}{|c|c|c|c|}
\hline \multicolumn{2}{|c|}{ Interprétation de V-oir } & Corpus V-oir ${ }_{\mathrm{TLF}}$ & Corpus V-oir $_{\text {W3 }}$ \\
\hline \multicolumn{2}{|c|}{ Lieu } & DORTOIR & GLANDOIR \\
\hline Instrument & $\begin{array}{c}\text { Artéfact } \\
\text { Partie du } \\
\text { corps }\end{array}$ & $\begin{array}{l}\text { GRATTOIR } \\
\text { MACHOIRE }\end{array}$ & $\begin{array}{l}\text { ENSACHOIR } \\
\text { RENIFLOIR }\end{array}$ \\
\hline \multicolumn{2}{|c|}{ Patient } & TIROIR, SAUTOIR & ASTICOTOIR, GRIFFOIR \\
\hline \multicolumn{2}{|c|}{ Cause } & REPOUSSOIR & TREBUCHOIR, ATTIROIR \\
\hline
\end{tabular}

Tab3 : Les interprétations construites par la règle morphologique RCL-OIR

\section{Corrélation entre $\mathbf{V}$ et les interprétations de $\mathbf{V}$-oir}

Le deuxième et principal résultat auquel nous aboutissons est l'ébauche de la règle de construction des V-oir : nous montrerons tout d'abord que l'interprétation du nom construit par la règle est sensible à la transitivité du verbe de base (section 4.1) et ensuite que, contrairement à ce qui a été mis en évidence pour d'autres types de lexèmes déverbaux, les V-oir ne s'identifient pas à un argument du verbe de base (section 4.2.2), même lorsque la structure argumentale de celui-ci comporte un élément remplissant un rôle instrumental ou locatif (section 4.2.1). 


\subsection{Propriétés de V}

\subsubsection{Aspect verbal}

Les verbes de base des déverbaux en -OIR incarnent des prédicats vérifiant tous les types aspectuels dynamiques.

[8] activités (PARLOIR, TROTTOIR, COMPTOIR, BEUGLOIR, TRAVAILLOIR, REGARDOIR),

[9] accomplissements (ALLUMOIR, DOROIR, ASSASSINOIR),

[10] achèvements (ETEIGNOIR, ARRIVOIR).

Seuls quelques statifs ont été recensés, à l'exception des verbes à localisation spatiale :

[11] AIMOIR, SOUFFROIR, VIVOTOIR

\subsubsection{Transitivité}

Les noms déverbaux en -OIR sont massivement construits sur des bases verbales transitives (au sens de (Levin and Rappaport Hovav, 1995), c'est-à-dire des verbes dont le premier argument correspond à un agent ou une cause et dont le deuxième correspond à un patient), comme en témoignent les statistiques effectuées sur le corpus du TLF ( $80 \%$ de bases verbales transitive) ou de La Toile (60\%): LAVOIR, APPELOIR.

Les autres bases verbales servant à la construction des noms en -OIR sont intransitives et relèvent de trois classes principales : celle des prédicats se réalisant en syntaxe accompagnés d'un argument de type SP à valeur locative, but, etc. (ECHAPPATOIRE, ALLOIR), celle des prédicats inergatifs, dont l'unique participant sémantique est agentif (DEGUEULOIR, RONFLOIR, TREMOUSSOIR) et enfin celle des prédicats inaccusatifs, dont l'unique participant sémantique est un patient, et comprenant notamment les verbes psychologiques et les verbes de mouvement à direction inhérente (GLISSOIR, $\underline{\text { ARRIVOIR, }} \underline{\text { SOUFFROIR). }}$

\subsubsection{Transitivité et interprétation instrumentale: surreprésentation, mais pas systématicité}

Comme l'avait déjà observé (Gamillscheg and Spitzer, 1921), et à l'instar de ce qui a été observé dans la formation des mots composés $\mathrm{VN}$, $\mathrm{NV}$ et des noms déverbaux en -eur, un verbe transitif réalisant un prédicat agentif affectant un patient (et de surcroît entraînant un changement d'état au moyen d'un instrument) est fréquemment la base d'un nom d'instrument $\left(80 \%\right.$ des $\mathrm{V}$-oir ${ }_{\mathrm{TLF}}$ et $63 \%$ des V-oir ${ }_{\mathrm{W} 3}$, selon la réussite aux batteries de tests présentés dans le tableau Tab1).

[12] DEMELOIR, « Peigne à dents très écartées servant à démêler les cheveux » (TLF)

[13] DECHIROIR, «j'ai vu que des guns comme la mp5 tiraient à plus de 1000 coups a la minute ... c pas interdit par la convention de genève ce genre de déchiroir automatique? Sinon cowboy c quoi tes sources ... » (La Toile, Yahoo 20/12/06).

[14] SOUDOIR, «Le souffleur d'air chaud professionel, soudoir, torch,... pour le professionnel, 5 fonctions en $1 »($ La Toile, Yahoo 20/12/06).

[15] DESENFUMOIR ... «Scully: "Mulder, je voulais te faire cadeau d'un desenfumoir pour noël mais je crois ça sera avancé : tes fumées ... » (La Toile, Yahoo 20/12/06).

Inversement, un verbe instransitif sert majoritairement de base à la construction d'un nom de lieu.

[16] TROTTOIR, «Passage surélevé établi pour la circulation des piétons d'un (ou des deux) côté(s) d'une rue, d'un pont, d'un quai... » (TLF) 
[19] BAVARDOIRE, «Un bavardoir (chat room) est un espace virtuel où des internautes se retrouvent pour bavarder sur un ... » (La Toile, Yahoo 20/12/06).

Nous pourrions expliquer cette relation entre transitivité/intransitivité du verbe et interprétation instrumentale/locative des V-oir en reprenant l'idée de (Gamillscheg and Spitzer, 1921) selon laquelle un verbe désignant un procès agentif et affectant un patient implique plus naturellement l'utilisation d'un instrument pour la réalisation de ce procès qu'un verbe décrivant une activité ne mettant pas en jeu un rapport agent/patient.

Néanmoins nos corpus montrent que cette implication n'est pas systématique. En effet, les verbes d'accomplissement ne servent pas uniquement de base à la construction de noms d'instrument, mais également de noms de lieu ([20], [21]); inversement, les noms d'instruments ne sont pas systématiquement construits sur des verbes transitifs mais aussi instransitifs ([22]) :

[20] CHAUFFOIR, «Spéc. Chauffoir public. Pièce chauffée que l'administration publique ouvre aux pauvres, en hiver. Le pavillon de gauche, au-dessus du chauffoir public (NERVAL, Bohème galante, 1853, p. 114).»

[21] ASSASSINOIR, " Quand ils les escortaient dans le lieu dit assassinoir, ils laissaient aux Arabes le soin de les piller et de les...»(La Toile, Yahoo 20/12/06).

MOUSSOIR, MIROIR, RASOIR

\subsection{Interprétation de V-oir et arguments logiques de V}

Nous avons vu (section 4.1) qu'une corrélation préférentielle peut être établie entre la transitivité du verbe de base et l'interprétation (lieu ou instrument) du nom dérivé. Nous examinons maintenant si cette interprétation est prédictible en fonction de la structure argumentale (ou sémantique) du verbe de base. Cette prédiction a en effet été vérifiée dans le cas de certaines RCL formatrices de déverbaux : par exemple, les noms formés par la RCL-eur correspondent préférentiellement à l'agent de V, quand celui-ci est agentif (DANSEUR), les exceptions à cette règle (NAISSEUR, TREBUCHEUR) correspondant à une interprétation perfective ou causative du verbe de base (NAISSEUR $=$ « celui qui fait naître ») (Fradin and Kerleroux, 2003a). Un autre exemple (Fradin, 2003) est celui des adjectifs déverbaux en -able, dont le nom modifié réfère prototypiquement ${ }^{9}$ au patient du verbe de base (PERISSABLE, MANGEABLE) où au site, quand $\mathrm{V}$ est un verbe de mouvement (CIRCULABLE, SKIABLE). On pourrait mentionner encore d'autres études (par exemple, les résultats dans (Villoing, 2003) ou (Fradin, 2005) concernant les noms composés VN) qui convergent vers le même résultat: l'interpétation du déverbal dépend des propriétés argumentales et sémantiques du verbe de base.

En conséquence il est légitime de se demander si une telle corrélation existe entre V-oir et le verbe de base : lieu et instrument peuvent-ils être des rôles prenant part à la structure sémantique d'un verbe donné ? Et, si oui, V-oir est-il dérivé d'un tel verbe avec l'interprétation (lieu / instrument) correspondant à cet argument? Afin de répondre à la première question (section 4.2.1) nous examinons certains travaux qui se sont intéressés aux lieux et instruments dans la structure argumentale des verbes. Nous confrontons ensuite nos données à ces résultats (section 4.2.2).

\subsubsection{Verbes sélectionnant un argument locatif ou instrumental}

Les rôles thématiques instrument et lieu ont rarement constitué l'objet central d'études syntaxiques. En effet, contrairement aux rôles agent et patient, lieu et instrument se réalisent le plus souvent sous la forme 
de syntagmes optionnels, et, de ce fait, sont relégués au rang d'ajout, n'intervenant pas dans la structure argumentale des verbes. Contrairement aux syntacticiens, les auteurs de sémantique lexicale examinent le rôle des participants logiques intervenant dans la décomposition de la structure sémantique d'un prédicat, indépendemment de leur réalisation syntaxique. Les sections 4.2.1.1 et 4.2.1.2 résument certains de ces travaux.

\subsubsection{Instrument}

Les rôles agent et instrument sont parfois vus dans la littérature comme les extrémités d'un continuum (cf. (Dressler, 1986)). Ainsi, dans le système-théta de T. Reinhart, présenté dans (Reinhart, 2002) la présence d'un rôle instrumental est subordonnée à l'attribution du trait 'cause' à l'argument externe d'un verbe $\mathrm{V}$ dans la grille théta de celui-ci. Ce trait, commun aux agents animés, aux instruments non-animés et aux forces non volitionnelles (Max/le marteau/l'orage a cassé la vitre) prédit l'alternance agent/instrument en position sujet de V (Max a cassé la vitre avec un marteau --> le marteau a cassé la vitre : cf. TEST1d à la section 3.1), ainsi que la corrélation entre présence d'un syntagme désignant l'instrument et existence d'un argument externe, éventuellement implicite (la vitre a été cassée avec un marteau). Puisque la présence d'un constituant instrument sous-entend celle d'un argument externe (implicite) et que, comme le soulignent entre autres (Fagan, 1992; Fellbaum, 1986; Keyser and Roeper, 1984), les V inaccusatifs ne tolèrent aucun argument externe (implicite), il en résulte que ces $\mathrm{V}$ sont incompatibles avec la présence d'un constituant instrumental (*la vitre a cassé avec un marteau). Le modèle de $\mathrm{T}$. Reinhart rend bien compte de ce fait qui conforte les conclusions obtenues à la section 4.1.

L'interchangeabilité entre agent et instrument explique comment un même type morphologique de nom (les noms construits par la RCL-VN ou par la RCL-eur) peut désigner soit l'agent du verbe V intervenant dans la construction du nom (un garde-barrière garde la barrière, un lecteur lit), soit l'instrument qui a servi à accomplir l'action décrite par V (un ouvre-boîte est l'instrument utilisé par un agent pour ouvrir les boîtes, un agrandisseur est l'appareil servant à faire des agrandissements), soit parfois les deux (un transporteur est à la fois l'individu et le véhicule destinés au transport de marchandises). Les RCL-eur et RCLVN, sélectionnent en effet un prédicat verbal V proto-agentif (Dowty, 1991) en position de base ou de constituant (voir (Fradin, 2005)). Plusieurs études (cf. (Booij, 1986; Cruse, 1973; Fillmore, 1968; Ulland, 1993) entre autres) ont montré qu'il est souvent difficile de distinguer agent et instrument qui se confondent parfois en ce que (Booij, 1986) nomme l'agent impersonnel. Pour (Croft, 1991 :185) et (Van Valin and LaPolla, $1997: 144$ ) (cités par (Fradin, 2005)), cette double interprétation sémantique est subsumée par un seul et même rôle proto-agentif : Actor.

Contrairement aux noms de type VN ou V-eur, les V-oir ne dénotent jamais l'agent de V (voir section 3.2.2), ce qui nous conduit à exclure cette piste : d'ailleurs, il ne suffit pas à $V$ d'être de type agentif pour servir de base à un V-oir désignant un instrument (ainsi, PARLER est agentif, et PARLOIR s'interprète comme un lieu).

Parmi les études de sémantique lexicale qui dissocient l'instrument de l'agent, celle de (Pustejovsky, 1995) et (Pustejovsky, 1996) ne s'intéresse pas spécifiquement pas aux rôles sémantiques, mais définit un cadre général pour la caractérisation des arguments logiques d'un prédicat ${ }^{10}$. L'auteur propose, dans le modèle formel du Lexique Génératif, de décomposer la structure argumentale d'un prédicat en trois types : les arguments vrais (réalisés obligatoirement en syntaxe), les arguments par défaut (réalisés optionnellement en syntaxe) et les arguments cachés (dont la réalisation en syntaxe est normalement impossible). Un argument caché code le participant logiquement nécessaire à l'accomplissement du procès décrit par le prédicat, mais dont l'expression est exclue car redondante: souvent, il s'agit de l'instrument (SCIE / SCIER : «je scie les planches ( $\square+*$ *avec une scie) »), mais pas toujours (PRISON / EMPRISONNER: "On a emprisonné le coupable $\left(\square+*\right.$ dans une prison) ») ${ }^{11}$. L'argument caché (en particulier quand il joue le rôle instrument) s'identifie donc le plus souvent avec le nom de base du verbe, ce qui fait que les verbes pouvant sélectionner un argument instrumental sont majoritairement dénominaux, construits par conversion ou par préfixation en $\mathrm{en}^{-{ }^{12}}$. Si nous adoptons cette analyse, cela suppose que V-oir désigne l'argument instrumental de V si l'expression «V avec DET V-oir» est 
redondante, en d'autres termes si V-oir constitue l'argument caché de V. Ceci, le plus souvent, sousentend que $\mathrm{V}$ est dénominal.

\subsubsection{Lieu}

Contrairement au rôle instrument, le rôle locatif peut correspondre à un complément obligatoire du verbe qui incarne toujours un prédicat de mouvement (eg. ALLER, METTRE), ou de localisation spatiale (eg. HABITER). La plupart de ces prédicats cependant se construisent en français avec un complément de lieu optionnel, souvent analysés en syntaxe comme des ajouts (pour une discussion, voir (Bonami, 1999)), ce qui nous a conduit, encore une fois, à nous tourner vers les travaux de sémantique lexicale pour identifier les types de verbes susceptibles de sélectionner un argument de type locatif. Il en résulte que la plupart des auteurs des études consultées analysent l'ensemble des types sémantiques de verbes de mouvement ou de localisation spatiale comme sélectionnant un argument de lieu. On pourra se référer, par exemple, à (Levin, 1993; Levin and Rappaport Hovav, 1995; Levin and Rappaport Hovav, 1999) pour des discussions concernant les verbes de mouvement à direction inhérente, les verbes de manière de mouvement et les verbes e localisation spatiale ; outre les travaux de (Levin, 1993; Pinker, 1989) et (Goldberg, 1995) déjà évoqués à la section 3.1.1, nous renvoyons à (Davis and Koenig, 2000; Davis, 2001; Ibañez Moreno and Ortigosa Pastor, 2004; Van Valin and LaPolla, 1997), pour une analyse de la structure sémantique des prédicats verbaux de mouvement causé.

\subsubsection{V-oir n'est pas construit sur un argument de $V$}

Les verbes qui sélectionnent un argument instrumental ou locatif étant identifiables, il nous reste à vérifier si nos données du TLF et de la Toile, dont l'interprétation (lieu/instrument) a été établie (cf. section 3.1), correspondent effectivement à l'argument (lieu/instrument) du verbe de base.

Nous notons tout d'abord que cette corrélation s'avère généralement fausse dans le cas des V-oir s'interprétant comme des instruments. En effet, ceux-ci ne jouent pas le rôle de l'argument instrumental d'un verbe $\mathrm{V}$ appartenant à l'un des types identifiés à la section 4.2.1.1. Considérons en effet les V-oir instrumentaux dont la base $\mathrm{V}$ est un verbe dénominal. D'après notre corpus, la base nominale de $\mathrm{V}$ s'interprète quasi-exclusivement comme une cible (PLATRE ${ }_{\text {CIBLE }}$ PLATRER: PLATROIR INSTR $_{\text {; }}$; FUMEE $_{\text {CIBLE }}$ /ENFUMER :ENFUMOIR INSTR). Dans de rares exceptions, V-oir désigne l'instrument qui a remplacé celui auquel le nom base de $\mathrm{V}$ fait référence : ainsi, HACHOIR (HACHER $<$ HACHE) et RAPOIR (RAPER < RAPE) désignent les instruments qui se substituent, respectivement, à HACHE et RAPE, dans l'exécution du procès décrit par $\mathrm{V}:$ on hache avec un hachoir (qui remplace la hache); on râpe avec un râpoir (à la place de la râpe).

En ce qui concerne, maintenant, la corrélation entre un V-oir locatif et la structure argumentale de V, elle est tout aussi improbable. Tout verbe $\mathrm{V}$ de mouvement sélectionné par la RCL-OIR ne produit pas nécessairement un V-oir de lieu (section 4.2.2.1). De même, tout V-oir de lieu n'a pas pour base un verbe $\mathrm{V}$ de mouvement (section 4.2.2.2). En d'autres termes, l'interprétation locative de V-oir et la classification sémantique de $\mathrm{V}$ parmi les prédicats de mouvement ne constituent des conditions ni nécessaires ni suffisantes à l'existence d'une relation morphologique entre $\mathrm{V}$ et V-oir. Les données chiffrées qui témoignent de cette observation sont réunies et illustrées dans le tableau Tab4, auquel renvoient les paragraphes ci-dessous.

\subsubsection{Verbe de mouvement et V-oir instrumental}

Nos observations nous conduisent à constater que les verbes de mouvement sont peu nombreux à servir de base à la dérivation en -OIR. Ils ne sont que 40, sur l'ensemble des 283 V-oir TLF $_{\text {(locatifs ou }}$ instrumentaux). Parmi ceux-ci, 23 (soit la moitié) seulement forment un nom locatif. Les proportions sont les même dans le corpus $\mathrm{V}-$-oir $_{\mathrm{W} 3}: 43$ verbes de mouvement (sur 304 noms en -OIR), dont la moitié (25) forment un nom à interprétation locative (cf. exemples 1a, 1b, 1c, Tab4: les exemples soulignés appartiennent à $\mathrm{V}$-oir $\left.\mathrm{W}_{\mathrm{W}}\right)$. Les 35 autres verbes de mouvement servent donc de base à la formation de noms d'instrument (cf. 2a, 2b, 2c, Tab4). Rappelons enfin que quelques verbes de mouvement constituent 
la base de noms en -OIR s'interprétant comme le patient (POUSSOIR, TIROIR) voire la cause (REPOUSSOIR, ATTIROIR) du prédicat verbal.

\subsubsection{V-oir locatif et verbe autre que de mouvement}

A l'inverse, les exemples du tableau Tab4 témoignent du fait que la majorité des noms en -OIR à interprétation locative (59/82 soit $72 \%$ pour TLF, $142 / 167$ soit $85 \%$ pour la Toile) ont comme base un verbe qui ne décrit ni un prédicat de mouvement, ni un prédicat de localisation spatiale. On trouve parmi ces verbes une quarantaine de prédicats intransitifs à sujet proto-patient (ex. 1e), l'immense majorité étant cependant constituée de prédicats à sujet proto-agentif (ex. 1d). L'absence de V-oir instrumental basé sur un prédicat verbal inaccusatif (ex. 2e) peut se voir comme une confirmation indirecte de l'attraction des V-oir instrumentaux pour les bases transitives (ce qui a été montré à la section 4.1).

\begin{tabular}{|c|c|c|c|c|c|}
\hline \multirow[t]{2}{*}{$\begin{array}{l}\text { Type de } \\
\text { verbe }\end{array}$} & \multirow[t]{2}{*}{$\begin{array}{c}\text { Nombre } \\
\text { (total : } \mathbf{5 8 7}\end{array}$} & \multicolumn{2}{|c|}{$\begin{array}{c}\text { V-oir de lieu : } 249\left(82_{\mathrm{TLF}}+\right. \\
\left.\underline{167}_{\mathrm{W} 3}\right)\end{array}$} & \multicolumn{2}{|c|}{$\begin{array}{c}\text { V-oir instrument : } 338 \text { (201 TLF } \\
\left.+\underline{137}_{\mathrm{W} 3}\right)\end{array}$} \\
\hline & & Exemples & Nombre & Exemples & Nombre \\
\hline $\begin{array}{l}\text { Prédicat de } \\
\text { localisation } \\
\text { spatiale ou } \\
\text { de manière } \\
\text { de } \\
\text { mouvement }\end{array}$ & \multirow[t]{3}{*}{$\begin{array}{l}83= \\
40_{\mathrm{TLF}}+\underline{43} \mathrm{~W} 3\end{array}$} & $\begin{array}{l}\text { (1a) PROMENOIR, } \\
\text { GAMBADOIR, } \\
\text { JUCHOIR, } \\
\text { AVACHISSOIR }\end{array}$ & \multirow[t]{3}{*}{$\begin{array}{l}48= \\
23_{\mathrm{TLF}}+\underline{25} \mathrm{~W} 3\end{array}$} & $\begin{array}{l}\text { (2a) NAGEOIRE, } \\
\text { PASSOIRE }\end{array}$ & \multirow[t]{3}{*}{$\begin{array}{l}25 \\
17_{\mathrm{TLF}}+\underline{18} \mathrm{~W} 3\end{array}=$} \\
\hline $\begin{array}{l}\text { Prédicat de } \\
\text { mouvement } \\
\text { à direction } \\
\text { inhérente }\end{array}$ & & $\begin{array}{l}\text { (1b) DEVALOIR, } \\
\text { BAISSOIR }\end{array}$ & & $\begin{array}{l}\text { (2b) MONTOIR }{ }^{13} \text {, } \\
\text { REMONTOIRE } \\
\text { DEPLAÇOIR }\end{array}$ & \\
\hline $\begin{array}{l}\text { Prédicat de } \\
\text { mouvement } \\
\text { causé }\end{array}$ & & (1c) DEVERSOIR & & $\begin{array}{l}(2 \mathrm{c}) \quad \text { LANÇOIR, } \\
\text { REPOUSSOIR, } \\
\text { POSOIR }^{\mathrm{v}}\end{array}$ & \\
\hline $\begin{array}{l}\text { Autres } \\
\text { types de } \\
\text { prédicats } \\
\text { (proto- } \\
\text { agentifs) }\end{array}$ & \multirow[t]{2}{*}{$\begin{array}{l}504= \\
243_{\mathrm{TLF}}+\underline{261} \mathrm{~W} 3\end{array}$} & $\begin{array}{l}\text { (1d) PARLOIR, } \\
\underline{\text { DEFEQUOIRE, }} \\
\underline{\text { BACHOTOIR }}\end{array}$ & \multirow[t]{2}{*}{$\begin{array}{l}201= \\
59_{\mathrm{TLF}}+\underline{142} \mathrm{~W} 3\end{array}$} & $\begin{array}{l}\text { (2d) DEMELOIR, } \\
\underline{\text { DECHIROIR, }} \\
\underline{\text { SOUDOIR }}\end{array}$ & \multirow[t]{2}{*}{$\begin{array}{l}303= \\
184_{\mathrm{TLF}}+\underline{119} \mathrm{~W} 3\end{array}$} \\
\hline $\begin{array}{l}\text { Autres } \\
\text { types de } \\
\text { prédicats } \\
\text { (proto- } \\
\text { patients) }\end{array}$ & & $\begin{array}{l}(1 \mathrm{e}) \quad \text { SALIVOIR, } \\
\text { MOUROIR, } \\
\text { PIONCCOIR, } \\
\text { ENRHUMOIR } \\
\end{array}$ & & $(2 \mathrm{e})$ & \\
\hline
\end{tabular}

Tab4 : Types de Vet interprétations des V-oir

\section{Conclusion}

L'étude que nous avons menée des noms déverbaux en -OIR du français a eu pour objectif de mettre au jour les relations que construit la règle morphologique entre l'interprétation de ces noms et les propriétés sémantiques, argumentales et syntaxiques du verbe de base. Nous voulions en particulier vérifier si cette relation était comparable à celle reconnue dans la formation d'autres noms déverbaux, à savoir (i) que la règle construit une ou deux interprétations préférentielles et, éventuellement, des interprétations périphériques, et (ii) qu'il existe une corrélation étroite et prédictible entre l'interprétation du dérivé et la 
structure argumentale du verbe de base. En outre, nous souhaitions vérifier si les conclusions auxquelles nous aboutissions seraient différenciées selon que les noms étudiés relevaient du corpus du TLF ou du corpus de néologismes extraits de La Toile, évaluant ainsi dans quelle mesure le registre de langue pouvait être un critère pertinent ou non pour l'étude.

Les résultats auxquels nous avons abouti sont les suivants :

(1) Nos conclusions sont identiques eu égard à l'origine des noms examinés et valent aussi bien pour les V-oir éventuellement "volatiles" issus de La Toile (créés à la volée, quel que soit le niveau de langue considéré, l'objectif discursif visé, le domaine textuel ou scientifique envisagé) que pour les V-oir trouvés dans le TLF, et à ce titre, appartenant au vocabulaire "commun" ou à une langue plus recherchée ou spécialisée ou encore désuète, obsolète, momifiée, fossilisée.

(2) Nos conclusions confirment la prédominance de deux interprétations pour les V-oir (lieu et instrument) au détriment d'autres significations très minoritaires (patient, cause).

(3) Contrairement aux autres déverbaux dont l'étude est rappelée dans cet article, la RCL-oir n'associe pas V-oir à l'un des éléments de la structure argumentale de V, ni à aucune autre de ses caractéristiques sémantiques (aspect, décomposition logique, classe sémantique). A l'inverse, cette RCL met en œuvre deux contraintes :

- le dérivé V-oir s'identifie à un participant au scénario impliqué par le prédicat verbal de base (le lieu ou l'instrument) qui n'est pas prévu dans la structure argumentale de celui-ci : on y reconnaît un ajout syntaxique.

- le type sémantique de cet ajout est corrélé à la transitivité de $\mathrm{V}$ : les verbes transitifs privilégient la modification par un ajout instrumental, alors que les verbes intransitifs servent préférentiellement de base à des V-oir modifieurs locatifs.

Nos résultats nous conduisent désormais à orienter notre travail vers trois directions principales :

(1) Elargissement du corpus et généralisation de la règle

Une étude complète de la formation des déverbaux en -OIR requiert de prendre également en compte les adjectifs suffixés en -OIR (e.g. COMPENSATOIRE, VEXATOIRE, DINATOIRE) de façon à pouvoir déterminer s'il s'agit, en synchronie, de la même RCL. Si c'est le cas, nous devrons nous demander comment s'effectue la sélection de la base verbale : en particulier, qu'est ce qui facilite ou, au contraire, inhibe le choix d'un même verbe dans la formation des dérivés nominal et adjectival (e.g. NAGEOIRE, NATATOIRE) ?

(2) Transitivité des verbes de base à V-oir

La deuxième question touche à l'explication de la transitivité des verbes de base dans la formation de V-oir : le rapport entre verbes $\mathrm{V}$ intransitifs et transitifs servant de base aux V-oir est-il différent du rapport entre $\mathrm{V}$ intransitifs et transitifs du lexique général ? Une réponse négative sous-entendrait que les limites de la productivité de la RCL-oir ne sont pas à imputer à la valeur du verbe en input.

(3) Corrélation entre type sémantique du verbe de base et interprétation des noms dérivés

Enfin, la troisième question est liée à l'indépendance du type sémantique du verbe de base avec l'interprétation du nom dérivé. Cette observation peut conduire à s'interroger, en retour, sur le bien-fondé d'une telle corrélation, quand elle a été posée pour d'autres dérivés déverbaux.

\section{Références bibliographiques}

Alexiadou, A. (2001). Functional structure in nominals: nominalization, and ergativity. Amsterdam: John Benjamins.

Alexiadou, A. \& Haegeman, L. (2007). Noun Phrase in the Generative perspective: Studies in generative grammar, 71. Berlin: Walter de Gruyter. 
Bonami, O. (1999). Les constructions du verbe: le cas des groupes prépositionnels argumentaux - Analyse syntaxique, sémantique et lexicale, UFR de Linguistique, Université Paris 7: Thèse de doctorat.

Booij, G. (1986). Form and Meaning in Morphology: the case of Dutch "agent-nouns". Linguistics 24:503-518.

Booij, G. \& Van Haften, T. (1988). La syntaxe externe des mots dérivés Lexique 7:101-120.

Booij, G. (1992). Morphology, semantics and argument structure. In Thematic structure: its role in grammar, ed. I. M. Roca, Dordrecht: Foris, 47-64.

Boons, J.-P., Guillet, A. \& Leclère, C. (1976). La structure des phrases simples en français: constructions intransitives. Genève: Droz.

Busa, F. (1997). The Semantics of Agentive Nominals in the Generative Lexicon. In Predicative Forms in Natural Language, ed. P. Saint-Dizier, Amsterdam: Kluwer.

Cadiot, P. (1990). La préposition avec: grammaire et représentation. Le français moderne 58:152-173.

Cadiot, P. (1991). Ala hache ou avec la hache? Représentation mentale, expérience située et donation du référent. Langue Française 91:7-23.

Corbin, D. (1987). Morphologie dérivationnelle et structuration du lexique. Lille: Presses Universitaires de Lille.

Corbin, D. (1990). Associativité et stratification dans la représentation des mots construits. In Contemporary Morphology, eds. W. U. Dressler, H. C. Luschützky, O. E. Pfeiffer \& J. R. Rennison, Berlin - New York: Mouton de Gruyter, 43-59.

Corbin, D. (2004). French (Indo-European: Romance). . In An International Handbook on Inflection and Word Formation, eds. G. Booij, C. Lehmann \& J. Mugdan, New York: Mouton - Walter de Gruyter, 1285-1299.

Croft, W. (1991). Syntactic categories and grammatical relations. The cognitive organisation of information. Chicago: University of Chicago Press.

Cruse, D. A. (1973). Some thoughts on agentivity. Journal of Linguistics 9:11-23.

Dal, G. (1999). Suffixation par -ette et bases verbales Paper presented at Silexicales 2 : la morphologie des dérivés évaluatifs (2ème Forum de Morphologie, 29-30 avril 1999), Toulouse 37-48.

Davis, A. R. \& Koenig, J.-P. (2000). Linking as constraints on word classes in a hierachical lexicon. Language 76:56109.

Davis, A. R. (2001). Linking by types in the hierarchical lexicon. Stanford, CA: CSLI Publications.

de Mulder, W. \& Flaux, N. (2005). La préposition sur: essai d'analyse. In Prépositions et conjonctions de subordination: syntaxe et sémantique, ed. M. Tenchea, Timisoara: Excelsior Art, 87-102.

Dendale, P. \& de Mulder, W. (1998). Sur sur. Réflexions sur l'emploi des 'ressemblances de famille' en linguistique. Paper presented at Atti del XXIe congresso internazionale di linguistica e filologia romanza, Palermo:213-225.

Dowty, D. R. (1991). Thematic proto-roles and argument selection. Language 67:547-619.

Dressler, W. U. (1986). Explanation in natural morphology, illustrated with comparative and agent-noun formation. Linguistics 24:519-548.

Dubois, J. (1962). Etude sur la dérivation suffixale en Français moderne et contemporain. Paris: Larousse.

Fagan, S. (1992). The syntax and semantics of middle constructions: a study wich special reference to German. Cambridge: Cambridge University Press.

Fellbaum, C. (1986). On the middle construction in English. Bloomington: Indiana University.

Fillmore, C. (1968). The case for case. In Universals in Linguistic Theory, eds. E. Bach \& R. Harms, New-York: Holt, Rinehart, and Winston, 1-88.

Flaux, N. \& Van de Velde, D. (2000). Les noms en français: esquisse de classement. Paris: Ophrys.

Fradin, B. (2003). Nouvelles approches en morphologie. Paris: Presses Universitaires de France. 
Fradin, B. \& Kerleroux, F. (2003a). Quelles bases pour les procédés de la morphologie constructionnelle ? In Silexicales 3 : les unités morphologiques, eds. B. Fradin et al., Villeneuve d'Ascq: Presses Universitaires du Septentrion, 76-84.

Fradin, B. \& Kerleroux, F. (2003b). Troubles with lexemes In Topics in Morphology. Selected papers from the Third Mediterranean Morphology Meeting (Barcelona, September 20-22, 2001), eds. G. Booij, J. De Cesaris, S. Scalise \& A. Ralli, Barcelona: IULA-Universitat Pompeu Fabra, 177-196.

Fradin, B. (2005). On a semantically grounded difference between derivation and compounding. In Morphology and its Demarcations, eds. W. U. Dressler, D. Katovsky \& F. Rainer, Amsterdam/Philadelphia: John Benjamins, 161-182.

Gamillscheg, E. \& Spitzer, L. (1921). Beiträge zur Romanischen Wortbildungslehre: Linguistica. Genève: Léo S. Olschki.

Goldberg, A. (1995). Constructions: a construction grammar approach to argument structure. Chicago: Chicago University Press.

Grimshaw, J. (1990). Argument Structure. Cambridge (MA) / London: MIT Press.

Gross, M. (1975). Méthodes en Syntaxe. Paris: Hermann.

Gross, M. \& Kiefer, F. (1995). La structure événementielle des substantifs. Folia linguistica historica 16:43-65.

Hathout, N., Plénat, M. \& Tanguy, L. (2003). Enquête sur les dérivés en -able. In Cahiers de Grammaire, eds. N. Hathout, M. Roché \& N. Serna, Toulouse: ERSS, 49-91.

Ibañez Moreno, A. \& Ortigosa Pastor, A. (2004). A Semantic Typology of Causative Accomplishment Movement Verbs and their Argument-Adjuncts in Role and Reference Grammar. ATLANTIS 26:35-49.

Kerleroux, F. (2004). Sur quels objets portent les opérations morphologiques de construction ? In Lexique 16, ed. P. Corbin, Villeneuve d'Ascq: Presses Universitaires du Septentrion, 85-124.

Keyser, S. J. \& Roeper, T. (1984). On the Middle and Ergative Constructions in English. Linguistic Inquiry 15:381416.

Levin, B. (1993). English Verbs Classes and Alternations. Chicago, IL: University of Chicago Press.

Levin, B. \& Rappaport Hovav, M. (1995). Unaccusativity. Cambridge, MA: MIT Press.

Levin, B. \& Rappaport Hovav, M. (1999). Two structures for compositionally derived events. Proceedings of SALT 9:199-223.

Maienborn, C. (2003). Event-internal modifiers: Semantic underspecification and conceptual interpretation. In Modifying Adjuncts, eds. E. Lang, C. Maienborn \& C. Fabricius-Hansen, Berlin / New York: Walter de Gruyter, 475-509.

Matthews, P. H. (1991). Morphology (1st edition : 1974). Cambridge: Cambridge University.

Meyer-Lübke, W. (1894). Grammatik der romanischen Sprachen. Leipzig: Reisland.

Milner, J.-C. (1982). Ordres et raisons de langue. Paris: Seuil.

Mithun, M. (1991). Active/agentive case marking and its motivations. Language 67:510-546.

Nyrop, K. (1899-1930). Grammaire Historique de la langue française vol. 3. Copenhague / Paris: Gyldendal / Nordisk Forlag.

Oshita, H. (1995). Compounds: A View from Suffixation and A-Structure Alteration. Yearbook of Morphology:179205.

Pinker, S. (1989). Learnability and cognition: the acquisition of argument structure. Cambridge, MA: MIT Press.

Plénat, M. (2005). Brèves remarques sur les déverbaux en -ette In La syntaxe au coeur de la grammaire, Recueil offert en hommage au 60ème anniversaire de Claude Muller, eds. F. Lambert \& H. Nølke, Rennes: Presses Universitaires de Rennes, 245-258.

Pustejovsky, J. (1995). The Generative Lexicon. Cambridge, MA: MIT Press. 
Pustejovsky, J. (1996). Lexical Shadowing and Argument Closure. Ms. Brandeis University, Boston, MA.

Reinhart, T. (2002). The theta-system: an overview. Theoretical Linguistics 28:229-290.

Roché, M. (2003). De la 'bonne formation' des dérivés en -on. Cahiers de Grammaire 28:91-112.

Sadler, L. \& Spencer, A. (1998). Morphology and argument structure. In Handbook of Morphology, eds. A. Spencer \& A. M. Zwicky, Malden, MA: Blackwell Publishings, 207-236.

Sammet, D. (1968). Die Substantivbildung mit Suffixen bei Chrestien de Troyes. Tübingen: Max Niemeyer Verlag.

Samvellian, P. (1995). Les nominalisations en français: arguments sémantiques et actants syntaxiques, UFR Linguistique Université Paris 7: doctorat.

Schmitz, A. \& Schmitz, B. (1995). Französische Wortbildung im 13. Jahrhundert Undersuchung der Substantivbildung bei Rutebeuf und Adenet le Roi. Bonn: Romanistischer Verlag.

Spencer, A. (1999). Transposition and argument structure. Yearbook of Morphology (1998):73-101.

Ulland, H. (1993). Les nominalisations agentives et instrumentales en français moderne. Berne: Peter Lang.

Van Valin, R. D. \& LaPolla, R. J. (1997). Syntax. Structure, meaning and function. Cambridge: Cambridge University Press.

Vandeloise, C. (1986). L'espace en français: sémantique des prépositions spatiales. Paris: Les éditions du Seuil.

Vandeloise, C. (2000). Plus de clarté dans la relation porteur/porté. In Traiani Augusti Vestigia Pressa sequamur, Studia Linguistica in honorem Lilianae Tasmowski, eds. M. Coene, W. de Mulder, P. Dendale \& Y. D'Hulst, Padova: Unipress, 753-769.

Villoing, F. (2003). Les bases des opérations de construction morphologique : des unités sémantiquement spécifiées. Illustration à la lumière de la composition [VN]N/A en français (3ème Forum de Morphologie, 20-21 septembre 2002, Lille) In Silexicales 3 : les unités morphologiques, eds. G. Dal, B. Fradin, F. Kerleroux, M. Plénat \& M. Roché, Villeneuve d'Ascq: Presses Universitaires du Septentrion, 213-219.

Williams, E. (1981). On the Notions 'Lexically Related' and 'Head of a Word. Linguistic Inquiry 12:245-274.

\footnotetext{
${ }^{1}$ Nous utiliserons la notation -OIR, pour subsumer les graphies -oir et -oire que prend l'exposant de la Règle de Construction de Lexème étudiée ici.

${ }^{2}$ Tout au long de l'article, les données issues du corpus de La Toile seront distinguées de celles issues du corpus du TLF par un soulignement.

${ }^{3}$ Suivant la convention initiée par Matthews (1991), on notera les lexèmes en petites capitales, et leur forme fléchie en minuscules italiques

4 « Système de réception de la farine et du son dans des sacs. »; « Corbeille dissymétrique pour ensacher à l'origine le charbon de bois » : La Toile, Yahoo 23/04/08

5 «Je peux regarder la tele du bord du lit, mais ce n'est pas la meme chose qu'une veritable glandoir de competition » : La Toile, Yahoo 23/04/08

${ }^{6}$ "Main artificielle avec laquelle, dans les hôtels des monnaies, on place les flans entre les deux coins, pour les frapper. » : La Toile, Yahoo 23/04/08

${ }^{7}$ « Moi je roule en sportive la semaine (xsara hdi 90) et en déplaçoir le weekend »: La Toile, Yahoo 23/04/08

8 « Le goblet plein déborde, et se vide dans l'écouloir prévu à cet effet » : La Toile, Yahoo 23/04/08

${ }^{9}$ Une étude basée sur l'examen de quantités massives de données de la Toile (Hathout, Plénat, \& Tanguy, 2003) montre les limites de cette prédiction. C'est pourquoi elle ne constitue qu'une tendance, ce que «prototypiquement » vise à exprimer.

${ }^{10}$ D'autres travaux de sémantique considèrent l'instrument comme un participant présent dans la structure du prédicat, à un niveau ou un autre. Ainsi C. Maienborn, dans (Maienborn, 2003) qui porte sur les prédicats de
} 
mouvement, et qui se consacre aux modifieurs événementiels, fait intervenir l'instrument comme composant sémantique dans la structure conceptuelle (CS) possible d'un prédicat. Elle distingue notamment, deux types de prédicats verbaux de mouvement: les prédicats de mouvement extrinsèque et les prédicats de mouvement intrinsèque. La structure conceptuelle (CS) des premiers incluent un argument supplémentaire qui joue le rôle d'instrument (et qui désigne le moyen de locomotion utilisé pour le déplacement); par exemple la CS de DRIVE comporte un instrument de type véhicule à moteur; à l'inverse, l'instrument intervenant dans la CS des seconds correspond à l'une des parties du corps (pex les pieds) de l'agent à l'origine du mouvement (pex MARCHER).

${ }^{11}$ Bien sûr, l'argument caché est réalisé lorsque son instanciation remet en cause cette redondance. Par exemple, quand l'expression de cet argument spécifie la valeur cachée: "je scie les planches avec une scie électrique ", ou désigne une entité ayant une fonction similaire à l'objet dénoté par l'argument caché: «je scie les planches avec un couteau de cuisine ».

12 Certes cette condition n'est pas absolument nécessaire. Certains prédicats non dénominaux comportent un argument caché dans leur structure argumentale. On pourrait admettre par exemple que l'accomplissement du procès décrit par GIFLER, nécessite l'usage d'un instrument obligatoire qui est, par défaut, la partie du corps MAIN de l'agent (" Bob a giflé Marie ( $\square+$ *avec sa main + avec ses deux mains + avec une pelle) »).

${ }^{13}$ « Grosse pierre, billot de bois qui permettait au cavalier de monter plus aisément à cheval. » (TLF)

${ }^{14}$ «Dispositif au moyen duquel on remonte un mécanisme d'horlogerie sans l'aide d'une clé. Montre à remontoir » (TLF) 